\title{
Magnetic fields in A stars
}

\author{
David Moss \\ Department of Mathematics, University of Manchester, Manchester M13 9PL, UK \\ email: moss@ma.man.ac.uk
}

\begin{abstract}
Some theoretical problems associated with the presence of large-scale magnetic fields in A stars are reviewed. Possible implications of some recent theoretical and observational results are discussed, with particular attention to the survival of fields from the ISM and their longterm stability. A more coherent picture of the origin of the strong large-scale fields seen in the magnetic CP stars may be beginning to emerge.
\end{abstract}

Keywords. Stars: magnetic fields, MHD

\section{Introduction}

After several decades of effort by astrophysicists, the strong large-scale magnetic fields seen in a minority of near-Main Sequence A-stars continue to present a number of problems. Although this paper (as the meeting) explicitly addresses A-type stars, most of what I write will apply also to the fields of the magnetic Bp stars, i.e., to all the magnetic CP stars. In this short paper I will not attempt to give yet another comprehensive review of the current situation (but see, e.g., Mestel 2003a,b, Moss 2001, 2003a for recent accounts), but rather I will focus on a few issues where I feel that some progress has been made in the last year or two.

A rather personal viewpoint leads to the following:

'Fundamental problems'

- * Origin of large-scale fields.

- The evolutionary status of the magnetic A-stars.

- *The long-term stability and survival of large-scale magnetic fields in stars.

- The ' $10 \%$ ' problem, why are only about $10 \%$ of Main Sequence A-stars observably magnetic?

'Second tier problems'

- *The striking difference in distributions of the obliquity angle between the rotational and magnetic axes between slower and faster rotators (Landstreet \& Mathys 2000).

- Why do apparently similar stars sometimes have quite different magnetic properties (including, e.g., close binary systems where both members are A stars)? Perhaps this is really part of the ' $10 \%$ problem' mentioned above.

'Third tier'

- The relatively slow rotation (as a class) of the magnetic A stars, Stepien (2000) suggests a plausible scenario involving the interaction of the field with a protostellar disc.

- The compositional anomalies and their relationship to the magnetic fields. An associated question, sharpened now by Aurière et al. (2004), is that all Ap and Bp stars appear to have surface fields in excess of about $100 \mathrm{G}$, and that no normal A stars are seen with fields $\lesssim 100 \mathrm{G}$ (down to the observational limit of $\mathrm{O}(10) \mathrm{G}$.

- The roAp phenomenon.

This is not intended to suggest any hierarchy of interest or difficulty, rather some sort of structuring, filtered by my personal interests. Below, I will concentrate mostly on the items above that are preceeded by an asterisk. 


\section{Origin of the fields}

The longstanding debate between fossil and dynamo origins of the fields is still undetermined, although the possibility of a 'hybrid' field, essentially a relic from a pre-Main Sequence dynamo, and more recently, a field resulting from a magnetorotational instability in the radiative envelope (Rüdiger et al. 2001, Arlt et al. 2005) have also been discussed. (It is of some interest to note that both of the latter would also appear as fossil relics from an earlier evolutionary phase.) In brief, the convective cores of Main Sequence A stars appear to be suitable sites for dynamo action. The dynamo theory for the magnetic A stars suggests that this dynamo generates the field that is observed at their surfaces. It is certainly to be expected that dynamos do operate in the convective cores of A stars (e.g., Browning et al. 2005). However, given the overlying deep radiative envelopes, any core-generated dynamo field has to rise passively from the core to the surface, the rise being mediated by buoyancy, compositional gradients, etc. Whilst arguments have been made lately that such a mechanism may be relevant in bringing some field to the surface, especially in stars rather more massive than being considered here, quite general arguments suggest that fields that are steady in the rotating frame and have the strength and spatial coherence of the observed fields are unlikely to be produced in such a manner (see Moss 1989, 2001, also McDonald \& Mullan 2004). Also why all, or nearly all, A stars are not observably magnetic, and the lack of correlation between magnetic and other properties, in particular angular velocity, remain difficult to explain with either core-dynamo or envelope magneto-rotational instability theories.

In contrast, the fossil theory proposes that the field seen on these stars on the Main Sequence is a relic of the field present in the ISM at the time of star formation, and subsequently amplified during contraction. This allows the 'initial conditions' of the ISM as an additional degree of freedom. There seems little doubt that there is 'enough' magnetic flux present in the pre-stellar material (see below). Nevertheless, this picture does encounter various problems, some of which will now be discussed.

Since the work of Hayashi and collaborators in the early 1960s, it has largely been believed that lower and middle Main Sequence stars initially contract down almost vertical paths in the Hertzsprung Russell diagram, where they are wholly or largely convective, see the broken part of the path in the schematic Fig. 1. Kinematically, such turbulence will distort the field, reducing its length-scale down to something like a diffusion scale, with consequent field expulsion or decay on a timescale very much shorter than a contraction time. Dynamically, general arguments and simulations suggest that the turbulence can concentrate the field into ropes, of strength about or rather larger than the equipartition strength, and these ropes may then be able to resist further shredding and decay. It can then be argued reasonably plausibly that significant signed flux can survive until a radiative core begins to form, at which time the flux ropes will become anchored in a stable region. As this radiative core grows, the field will diffuse to a more uniform geometry.

Quantification of such a scenario is difficult: it cannot be treated analytically, and it is still beyond any realistic simulation (the situation may change in the next few years). Moss $(2003 \mathrm{a}, \mathrm{b})$ constructed an order of magnitude model to describe the contraction of a magnetic star down a Hayashi track, concluding that if the survival in strong ropes idea is valid, more massive stars may be able to retain stronger fields to the Main Sequence than stars of about solar mass, but with a smooth dependence on mass that is unlike the turning on of the magnetic CP star phenomenon at $M \gtrsim 2 M_{\odot}$. The lack of evidence for a field in the solar radiative core of more than $\mathrm{O}(1) \mathrm{G}$ argues against the presence of a significant convective envelope on the Main Sequence being a decisive factor. 


\subsection{A more recent view of pre-Main Sequence stellar evolution}

Palla \& Stahler (1993, see also Marconi \& Palla 2005) published a model of pre-Main Sequence evolution in which more massive stars do not necessarily experience a largely convective phase. Their initial condition, rather than being an isolated gas sphere which first found equilibrium at the top of the Hayashi track, was a sphere embedded in an accreting envelope, such as might be expected from the late stages of the transition from a more-or-less freely falling gas cloud to a 'star' in dynamical equilibrium. Effectively, this envelope changes the outer boundary condition in such a way that stars of mass more than about $2 M_{\odot}$ (depending somewhat on choice of parameters) start their contraction as radiative objects along the almost horizontal part of the evolutionary track (solid in Fig. 1). Thus they do not experience an episode when they are largely or wholly convective. Interestingly, this is essentially the 'Henyey track', which pre-dated Hayashi's work (see, e.g., Schwarzschild 1958).

If this picture is valid, it provides a clear cut distinction between stars with $M \lesssim 2 M_{\odot}$, which experience large-scale turbulence and subsequent field destruction, and so do not appear as strongly magnetic on the Main Sequence, and those with $M \gtrsim 2 M_{\odot}$, which do not suffer this field destruction and so can retain their primordial fields. Nevertheless, this still leaves outstanding problems about the survival of flux from the ISM, especially the dynamical stability of large-scale field structures, why observed fields are all nonaxisymmetric (i.e., the field axis and rotation vector are non-parallel), and the $10 \%$ problem. These are all discussed, briefly at least, in the following sections.

\section{Nonaxisymmetric fields}

In general, the gravitational contraction of an initially rotating gas sphere can be expected to result in a state of differential rotation. Then, from the winding up of nonaxisymmetric field lines and the bringing together of field lines of opposite sign, enhanced decay can be expected to result (Fig. 2). This does not happen to axisymmetric fields, and so nonaxisymmetric fields are discriminated against (e.g., Rädler 1986). This is a purely kinematic argument. When dynamical effects are taken into account, the picture may change. The azimuthal stretching of field lines results in an azimuthal component of Lorentz force which acts to reduce the differential rotation (e.g., Mestel 2003a). Thus a strong enough nonaxisymmetric field can survive and dominate differential rotation. Rädler (1986) showed that, ignoring dynamical effects, field winding will be limited by ohmic dissipation after $n_{c}$ windings, where $n_{\mathrm{c}} \sim\left(\Delta \Omega L^{2} / 2 \pi \eta\right)^{1 / 3}$, i.e. after a time $t_{\mathrm{w}} \sim 2 \pi n_{\mathrm{c}} / \Delta \Omega$. If a typical dynamical timescale is $t_{\mathrm{D}} \sim L / v_{\mathrm{A}}$, with $v_{\mathrm{A}}$ the Alfvèn velocity, where $\Delta \Omega$ is an estimate of the variation in angular velocity $\Omega, L$ a length-scale for the field and $\eta$ is magnetic diffusivity in units of $\mathrm{cm}^{2} \mathrm{~s}^{-1}$, then the condition $t_{\mathrm{w}} \approx t_{\mathrm{D}}$ defines a field $B_{c} \sim \rho^{1 / 2}\left(\operatorname{L} \eta(\Delta \Omega)^{2}\right)^{1 / 3} \mathrm{G}$ (Moss 1992). This can be rewritten as

$$
B_{\mathrm{c}} \sim 4 \times 10^{4} \rho^{\frac{1}{2}}\left(\frac{\Omega}{10 \Omega_{\odot}}\right)^{\frac{2}{3}}\left(\frac{R}{3 R_{\odot}}\right)^{\frac{1}{3}}\left(\frac{\eta}{10^{12}}\right)^{\frac{1}{3}}\left(\frac{\Delta \Omega}{\Omega}\right)^{\frac{2}{3}}\left(\frac{L}{R}\right)^{\frac{1}{3}} \mathrm{G},
$$

and fields in excess of $B_{\mathrm{c}}$ can be expected to reduce rotational shear. $\rho$ is a typical density (in $\mathrm{cm}^{-3}$ ) and $\Delta \Omega / \Omega$ is an estimate of the fractional differential rotation. Putting $\rho=10^{-1} \mathrm{gm} \mathrm{cm}^{-3}, \Delta \Omega / \Omega=1, L \sim R, \eta=10^{9} \mathrm{~cm}^{2} \mathrm{~s}^{-1}, \Omega=10 \Omega_{\odot}$ gives $B_{\mathrm{c}} \sim 250$ $\mathrm{G}$, suggesting that nonaxisymmetric fields of moderate strength might survive and even control differential rotation. However, strong differential rotation and nonaxisymmetric fields cannot coexist.

We can make an experiment by 'rewinding' a kilogauss field in a Main Sequence star of about $3 M_{\odot}$ back through the pre-Main Sequence contraction. This value of the mean 
Table 1. Mean field strengths $\left\langle B_{1}\right\rangle$ and $\left\langle B_{2}\right\rangle$ (defined in text) for a $3 M_{\odot}$ star along its pre-Main Sequence evolutionary path, assuming flux freezing with $B \propto R^{-2}$. $B_{\mathrm{c} 1}$ is given by Eq. 3.1, with $\eta=10^{9} \mathrm{~cm}^{2} \mathrm{~s}^{-1}$, whereas for $B_{\mathrm{c} 2}, \eta=10^{12} \mathrm{~cm}^{2} \mathrm{~s}^{-1}$ when the star is largely convective $(\mathrm{C})$, and $\eta=10^{6} \mathrm{~cm}^{2} \mathrm{~s}^{-1}$ where it is radiative (R). $\Omega=10 \Omega_{\odot}, L=R, \Delta \Omega / \Omega=0.1$. All magnetic fields are in $\mathrm{G}$, and the mean density $<\rho>$ is in $\mathrm{cm}^{-3}$

\begin{tabular}{lccccc}
\hline$R / R_{\odot}$ & $\left\langle B_{1}\right\rangle$ & $\left\langle B_{2}\right\rangle$ & $\langle\rho\rangle$ & $B_{\mathrm{c} 1}$ & $B_{\mathrm{c} 2}$ \\
\hline $2(\mathrm{R})$ & $10^{3}$ & $2 \times 10^{4}$ & 0.5 & 500 & 50 \\
$5(\mathrm{R})$ & 160 & $3.2 \times 10^{3}$ & 0.03 & 200 & 20 \\
$10(\mathrm{C})$ & 40 & 800 & 0.004 & 80 & 800 \\
$20(\mathrm{C})$ & 10 & 200 & 0.0005 & 40 & 400 \\
\hline
\end{tabular}

field strength, referred to as $\left\langle B_{1}\right\rangle$, is proportional to $R^{-2}$ assuming flux conservation, see Table 1. An alternative viewpoint is to take an estimate of field strength at the top of the Hayashi track, as suggested by star formation models with approximate flux freezing after reionization (Desch \& Mouschovias 2001, Nakano et al. 2002). Moss (2003b, Table 1) estimated this to give a field of about $200 \mathrm{G}$ there, although the figure is rather uncertain (and some flux loss can certainly be expected to occur during the subsequent contraction process). This field, also proportional to $R^{-2}$ assuming flux conservation, is denoted by $<B_{2}>$. Table 1 also lists values $B_{\mathrm{c} 1}$ and $B_{\mathrm{c} 2}$, calculated from Eq. (3.1): $B_{\mathrm{c} 1}$ is given using $\eta=10^{9} \mathrm{~cm}^{2} \mathrm{~s}^{-1}$ throughout, whereas for $B_{\mathrm{c} 2}, \eta=10^{12} \mathrm{~cm}^{2} \mathrm{~s}^{-1}$ on the convective part of the track $\left(R \gtrsim 5 R_{\odot}\right)$ and $10^{6} \mathrm{~cm}^{2} \mathrm{~s}^{-1}$ on the radiative part $\left(R \lesssim 5 R_{\odot}\right)$. $<\rho>$ is a mean density. In this Table, $\left\langle B_{2}>\right.$ exceeds $B_{\mathrm{c} 1,2}$ everywhere, whereas $\left\langle B_{1}\right\rangle$ is of the order of, or somewhat smaller, than, $B_{\mathrm{c} 1}$, and much less than $B_{\mathrm{c} 2}$ when $R>5 R_{\odot}$.

Thus it appears that with 'turbulent' values of $\eta\left(\gtrsim 10^{12} \mathrm{~cm}^{2} \mathrm{~s}^{-1}\right.$ ? $)$, the field $<B_{2}>$ is about of the order of magnitude necessary to control differential rotation, so preventing excessive winding and enhanced decay, but that the field $\left\langle B_{1}\right\rangle$ is too small. Note that $\left\langle B_{1}\right\rangle$ is likely to be something of an underestimate, since some flux loss is probably unavoidable. But with values of the diffusivity appropriate to a radiative region $\left(\eta \lesssim 10^{6}\right)$, both these fields are large enough to limit differential rotation. Thus stars whose pre-Main Sequence evolution is completely along a radiative track might be expected to retain their large-scale nonaxisymmetric fields, with near-uniform rotation.

In passing, note that if large-scale fields of the strength discussed here were to be present thoughout the radiative part of the pre-Main Sequence star, then the magnetorotational instability discussed by Rüdiger et al. (2001) and Arlt et al. (2005) would be inhibited.

\section{Dynamical stability of large-scale fields}

Since the work of Tayler (1973), Markey \& Tayler (1973) and Wright (1973) it has been known that purely poloidal and purely toroidal fields in stars will be dynamically unstable. Essentially, poloidal fields near the neutral line, and toroidal fields near their axis have the topology of the $z$-pinch, which is subject to the kink instability (see e.g., Fig. 1 of Tayler 1973). This instability will reduce field length scales, presumably resulting in a greatly enhanced dissipation rate. It was shown that that a field topology with linked poloidal and toroidal field lines has improved stability properties, analogous to the stabilization of the $z$-pinch by a longitudinal field. The basic arguments are mostly simply presented in terms of axisymmetric fields, but the instability depends only on general properties of the field and its geometry (and is independent of field strength), and so is not restricted to axisymmetric fields. Rotation, especially when combined with nonaxisymmetric fields, and finite resistivity are further complicating features (see, e.g., 


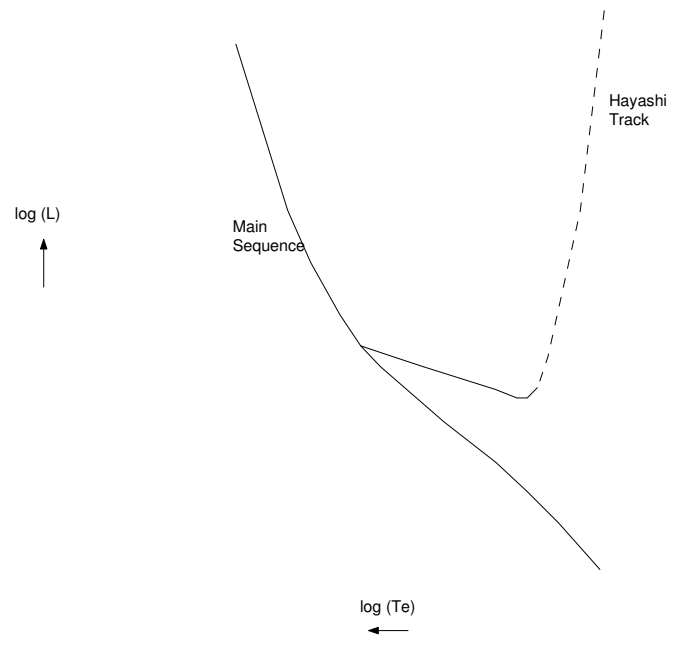

Figure 1. A schematic Hertzsprung Russell diagram, showing the Main Sequence and a pre-Main Sequence evolutionary track for a star of several solar masses. The largely convective Hayashi track is shown as a broken curve. According to Palla \& Stahler's work, such a star would only trace the radiative part of the track, shown as solid.

Pitts \& Tayler 1985). Given the difficulties of making further analytic progress it has been implicitly assumed that stable linked poloidal-toroidal configurations exist and are commonly found.

Very recently Braithwaite \& Spruit (2005) have simulated the dynamical evolution of the initial fields embedded in gas spheres. They find that dynamically stable magnetic fields with linked poloidal and toroidal fluxes naturally emerge, providing a very welcome confirmation for the picture described above, which had been widely accepted as plausible, but had lacked detailed confirmation. Moreover, depending on initial conditions, the fields may in some cases take about a classical diffusion time of $O\left(10^{9}\right)$ yr to reach the stellar surface (i.e., of order of or longer than the Main Sequence lifetime of A stars). This last figure is rather uncertain, as computationally some rescaling is necessary, and there is also a dependence on unknown initial conditions. Further, this timescale might be shortened by the effects of unconsidered (and uncertain) microinstabilities, which would lead to an effective enhanced diffusivity. The initial field strength remains a free parameter. Importantly, for these estimates to be relevant in the context of the magnetic A star problem, it seems that the star must be largely radiative (see Sect. 2.1 above).

\section{The angle between the rotation and magnetic axes}

Rather unexpectedly, Landstreet \& Mathys (2000) found that in the more slowly rotating magnetic $\mathrm{CP}$ stars $\left(P_{\text {rot }} \gtrsim 25 \mathrm{~d}\right)$ the angle $\beta$ between magnetic and rotation axes is small, whereas when $P_{\text {rot }} \lesssim 25 \mathrm{~d}$ it is large, with a significant fraction having $\beta$ near $90^{\circ}$. The origin of this distinctly nonrandom distribution is quite unclear.

Three mechanisms that could operate to modify an initial $\beta$-distribution have been identified. In an oblique rotator, magnetically controlled stellar winds exert both braking and precessional torques. Only rather idealized cases have been worked out in detail. These suggest that $\beta \rightarrow 0^{\circ}$ or $90^{\circ}$, depending on the surface field distribution (Mestel \& Selley 1970). This mechanism would have to operate before the arrival of the star on the Main Sequence.

Mestel et al. (1981) studied the motions resulting from the Eulerian nutation that occurs when $\beta \neq 0,90^{\circ}$, and deduced that the resulting dissipation of energy would 


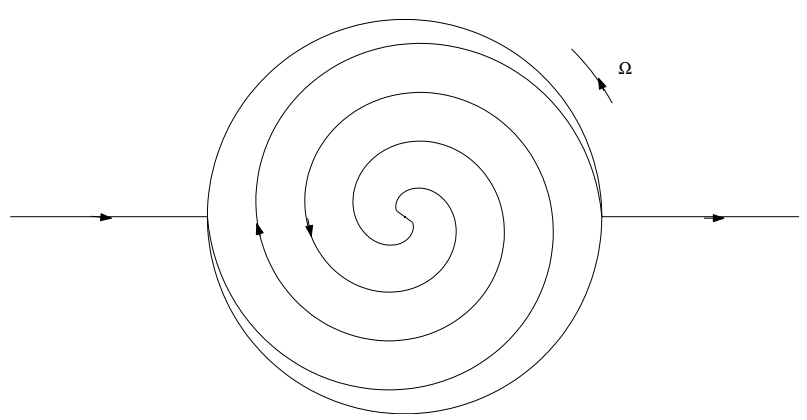

Figure 2. Cartoon, showing how an initial field line of small curvature lying perpendicular to the rotation axis will be wound up by a differential rotation, so that fields of opposite sense are brought close together, facilitating reconnection and decay. (Here, $d \Omega / d r<0$.)

again result in asymptotic states of $\beta=0^{\circ}$ or $90^{\circ}$, now depending on the position of the axis of maximum moment of inertia.

The timescales for both of these processes to operate depend on several ill-known factors, but it is conceivable that they could be relevant. The third mechanism is the advection of field by the rotationally driven (and magnetically modified) meridional circulation, which was shown by Moss $(1977,1984)$ to affect the observed angle $\beta$. This mechanism has the appealing property that for sufficiently rapid rotators, $\beta$ becomes smaller when the initial value of $\beta<55^{\circ}$, whereas for larger initial $\beta, \beta \rightarrow 90^{\circ}$. Rather disappointingly perhaps, it seems that the mechanism will only be effective for rotation periods of less than about $5 \mathrm{~d}$, and maybe substantially shorter.

Another, very speculative, possibility perhaps now emerges. Braithwaite \& Spruit's simulations do not take into account the effects of rotation. This can be argued to be unimportant if the Alfvèn travel time is shorter than the rotational period. For a $3 M_{\odot}, 5 R_{\odot}$ protostar, an internal field of $10^{5} \mathrm{G}$ has an Alfvèn time of order $25 \mathrm{~d} .10^{5} \mathrm{G}$ is rather a large field in the context of Table 1 , but $\left.\left\langle B_{1}\right\rangle,<B_{2}\right\rangle$ are mean poloidal fields, and toroidal fields that are comparable with or locally stronger than the poloidal fields will be present in stable configurations. Also it is plausibly an Alfvèn time for an interior region, where fields are larger than their mean values, that is relevant. Could the emergence of a stable field configuration result in different values of $\beta$ for faster and slower rotators? Again, it seems probable that only large-scale simulations will be able to answer this question.

\section{Conclusions}

It now seems possible to give a plausible broad-brush account of the history of the anomalously strong fields of the magnetic CP stars.

- If some (at least) stars follow the Palla \& Stahler (1993) pre-Main Sequence evolutionary history, then the presence of strong global-scale fields in a fraction of Main Sequence stars with $M \gtrsim 2 M_{\odot}$ can be explained, and also the absence of any such fields in less massive Main Sequence stars.

- Nonaxisymmetric fields of the strengths characteristic of magnetic CP stars are strong enough to resist winding by differential rotation, and indeed can bring about a state of near uniform rotation.

- Fields naturally evolve to dynamically stable configurations, which can survive for times as long as the Main Sequence lifetime of A stars. For most of this time these fields are likely to be predominantly dipolar at the surface. 
- It is possible that in some cases fields may emerge after a star reaches the Main Sequence - this can depend on a number of rather uncertain factors.

- It still seems to be necessary to appeal to variations in the initial conditions to resolve the ' $10 \%$ ' problem. There is now the additional feature that not only the total flux present in the protostellar medium, but also its spatial distribution, may play a role.

Crucially, this picture requires that the pre-Main Sequence evolution of the magnetic $\mathrm{CP}$ stars is substantially radiative, i.e., that global turbulent convection does not occur.

Of course, there are still a number of outstanding problems. Readers will have their own lists of priorities and concerns but I would like to emphasize again the question of the distribution of angles $\beta$ between magnetic and rotational axes (Sect. 5 ):

- Why do relatively rapid rotators $\left(P_{\text {rot }} \lesssim 25 \mathrm{~d}\right.$ ) have large values of $\beta$ (i.e. axes close to perpendicularity), whereas slower rotators have small $\beta$ and so axes that are more nearly aligned (Landstreet \& Mathys 2000)?

\section{Acknowledgements}

I am grateful to Leon Mestel for comments on a draft of this paper.

\section{References}

Aurière, M., Silvester, J., Wade, G.A. and 10 others 2004, preprint

Arlt, R. 2005, These Proceedings, 103

Braithwaite, H., Spruit, H. 2005, This meeting, http://www.ta3.sk/IAUS224/PDF/ET1.pdf

Browning, M. K., Brun, A. S., Toomre, J. 2005, These Proceedings, 149

Desch, S.J. \& Mouschovias, T. Ch. 2001, ApJ, 550, 314

Landstreet, J.D. \& Mathys, G. 2000, A\&A, 359, 213

McDonald, J. \& Mullan, D.J. 2004, MNRAS, 348, 702

Marconi, M., Palla, F. 2005, These Proceedings, 69

Mestel, L., 2003a, Stellar Magnetism (paperback edition, Clarendon Press; Oxford)

Mestel, L., 2003b, in: L.A. Balona, H. Henrichs \& T. Medupe (eds.), Magnetic fields in $O$, B and A stars: origin and connection to pulsation, rotation and mass loss, ASP Conf. Ser., 305 , p. 3

Mestel L. \& Selley, C.S. 1970, MNRAS, 149, 197

Mestel, L., Nittman, J., Wood, W.P., \& Wright, G.A.E., MNRAS, 195, 979

Moss, D. 1977, MNRAS, 178, 61

Moss, D. 1984, MNRAS, 209, 607

Moss, D. 1989, MNRAS, 236, 629

Moss, D. 1992, MNRAS, 257, 593

Moss, D. 2001, in: G. Mathys, S.K. Solanki, D.T. Wickramasinghe (eds.), Magnetic Fields across the HR Diagram, ASP Conf. Ser., 248, 305

Moss, D. 2003a, in: J. Arnaud \& N. Meunier (eds.), Magnetic activity of the Sun and stars, EAS Publ. Ser., Vol. 9, p. 21

Moss, D. 2003b, A\& A, 403, 693

Nakano, N., Nishi R. \& Umbeyashi T., 2002, ApJ, 573, 1999

Palla, F. \& Stalla, S.W., 1993, ApJ, 418, 414

Rädler, K.-H. 1986, in: Plasma Astrophysics, ESA, SP-251, p. 569

Rüdiger, G., Arlt, R. \& Hollerbach, R. 2001, in: G. Mathys, S.K. Solanki \& D.T. Wickramasinghe (eds.), Magnetic Fields across the HR Diagram, ASP Conf. Ser., 248, 315

Stępień, K. 2001, A\&A, 353, 227

Tayler, R.J. 1973, MNRAS, 161, 365

Markey, P. \& Tayler, R.J. 1973, MNRAS, 163, 77

Pitts, E. \& Tayler, R.J. 1985, MNRAS, 216, 139

Schwarschild, M. 1958, The Structure and Evolution of the Stars (Princeton University Press)

Wright, G.A.E., 1973, MNRAS, 162, 339 


\section{Discussion}

WADE: I want to stress the point that the $10 \%$ problem is not confined to the statistical incidence of fields in random, unrelated field stars. Rather, a more fundamental problem is that A stars which appear to be explicitly related (e.g., in open clusters and SB2 systems), and that have undergone similar/identical evolutionary scenarios, can have qualitatively different surface magnetic field characteristics (i.e., one is magnetic, one is not). This is a real puzzle!

Moss: I agree! I certainly did not intend to play down this aspect.

Piskunov: The two mechanisms of magnetic field generation, fossil and dynamo, must coexist in stellar interiors, at least during some stages of stellar evolution. Can we expect some significant interaction between the two fields? Can a fossil field affect the dynamo, or a dynamo field perturb a simple dipolar structure of the fossil field?

Moss: Turbulent convection in the core would by itself be expected to exclude the largescale field from the central regions, without any very large global effects. I cannot see any reason why a dynamo that generated relatively small-scale fields, as in the simulations of Browning et al. (2005), should change this significantly: it is hard to see how such a field in the core would reconnect to the global field. In reverse, the situation is less clear. I recently $(2004$, A\&A, 414, 1065) suggested that a field external to a dynamo with significant differential rotation could significantly affect the operation of the dynamo. Of course, someone should do the required simulations!

COWLEY: Regarding the question of whether a collapsing star is radiative or convective: is the primordial field itself strong enough to influence this question? To put this another way: if there is a field in the interstellar medium, is the star more likely to follow a radiative track than if there were no such field?

Moss: This problem was more-or-less the topic of my first published paper, in MNRAS in 1968! The result was that for lower mass stars the effect of a magnetic field, by slightly inhibiting convection, was to move the Hayashi track a small distance to the red. I would imagine that for more massive stars, and with contemporary ideas about pre-Main Sequence evolution, there would be at most a very marginal difference in the mass at which stars changed from having to not having a largely convective phase (see Sect. 2.1 above).

NoELS: I would like to point out that in the Henyey tracks the fully convective phase was suppressed because it was thought that this phase had no influence on the Main Sequence models. In the Henyey code (in the 1960s) the Hayashi track could be computed, but as computations took so long they were generally ignored and evolution was started as near to the Main Sequence as possible.

Moss: I was thinking of the paper by Henyey et al. (PASP 1955) where the possible effects of envelope convection are explicitly ignored. Hayashi (PASJ 1961) seems to have been the first to realise the dramatic effects of envelope convection on the pre-MS evolutionary tracks of lower mass stars. 